\section{COASTAL MARINE FISH BIODIVERSITY ALONG THE WESTERN COAST OF INDIA}

\section{Robert D. Sluka}

Chiltern Gateway Project, A Rocha UK, 18-19 Avenue Rd, Southall, Middlesex UB1 3BL, United Kingdom

bob.sluka@arocha.org

The commercial fish fauna of India has been studied extensively (James et al. 1994, 1996). However, little is known about noncommercial fishes (Kuthalingam et al. 1973, 1979; Sreenivasan \& Lazarus 1973; James et al. 1988; Murty 2002). Underwater visual censuses have been reported from Lakshadweep (Anand \& Pillai 2002, 2005), the Andaman and Nicobar Islands (Madhu \& Madhu 2007; Rajaram \& Nedumaran 2009) and from islands off the central western coast states of Goa and Karnataka (Sluka \& Lazarus 2004, 2005, 2006, 2009, 2010; Zacharia et al. 2008; Thomas et al. 2011). The present study reports on marine fish biodiversity studies completed on the south-west coast of India. This represents the first report of southwestern Indian fish biodiversity made using underwater visual observations. This report documents 184 species from this coast, 12 of which appear to be new records for India.

\section{Materials and Methods}

Data were collected by one observer (RDS) on SCUBA by recording observations on underwater paper and comparing to standard fish identification books (De Bruin et al. 1994; Allen 1997; Kuiter 1998). Observations were made on rocky substrata between Vizhinjam, Kerala State and Muttom, Tamil Nadu State (Image 1), January-
March 2002 ( $n=7$ sites and 30 hours sampling). Similar habitat was examined at Natrani Island, offshore of Murdeshwar, Karnataka State and Grand Island offshore of Panaji, Goa State (Image 1), SeptemberOctober $2002(\mathrm{n}=$ all surveys sum to

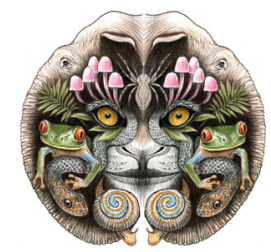

ISSN

Online 0974-7907 Print 0974-7893

\section{OPEN ACCESS} 8 and 13 hours of observation time, respectively, for each of these two sites). The habitat surveyed in Kerala and Tamil Nadu (depth range 3-30 m) was rocky with low coral cover $(<1 \%)$ (Sluka et al. 2012). The most abundant benthic colonizers were fine turf algae, encrusting sponges, barnacles and mussels. At Muttom, three types of habitats were surveyed and each considered a separate site: (i) shallow $(<10 \mathrm{~m})$, inshore ( $<500 \mathrm{~m}$ from shore), dead coral reef; (ii) offshore rocky islands ( $15 \mathrm{~m}$ depth); and (iii) submerged rocky habitat (30m depth). The habitat in western coast off Karnataka and Goa (depth range 2-12 m) was also rocky, but with higher coral cover (15\% and 31\% for Natrani and Grand Island, respectively). Other abundant benthic colonizers at these two sites were fine turfing algae, encrusting sponges, and polychaetes.

Species lists were developed and then checked for prior occurrences in India using Fishbase (www.fishbase. org) last updated on 26 April 2012. Other literature was also examined for occurrence records, including synonyms (Jones 1969; Murty 1969; Kuthalingam et al. 1973; Jones \& Kumaran 1980; Fischer \& Bianchi 1984; Murty et al. 1987; Allen 1991; Allen et al. 1998; Rao et al. 2000; Murty 2002; Suresh \& Thomas 2007).

\section{Results}

A total of 184 species from 41 families were recorded

DOI: http://dx.doi.org/10.11609/JoTT.03187.118 | ZooBank: urn:Isid:zoobank.org:pub:220D68BF-667B-4CF6-860D-010C7A76B047

Editor: J. Jerald Wilson, King Abdulaziz University, Jeddah, Saudi Arabia.

Date of publication: 26 January 2013 (online \& print)

Manuscript details: Ms \# 03187 | Received 30 April 2012 | Final received 05 October 2012 | Finally accepted 18 November 2012

Citation: Sluka R.D. (2013). Coastal marine fish biodiversity along the western coast of India. Journal of Threatened Taxa 5(1): 3574-3579; doi:10.11609/JoTT. 03187.118

Copyright: @ Sluka 2013. Creative Commons Attribution 3.0 Unported License. JoTT allows unrestricted use of this article in any medium, reproduction and distribution by providing adequate credit to the authors and the source of publication.

Funding: This study was funded by the Committee for Research and Exploration, National Geographic Society (Grant 7094-01).

Competing Interest: None.

Acknowledgements: We thank S. Thomas, F.G. Nazareth and V. Charloo for diving assistance. V. Charloo and K. Gregory of Barracuda Diving Services, Goa provided dive support at cost at Murdeshwar and Panaji. G. Anita Mary provided assistance in the field. R. Kuiter assisted with identifying several species. The author thanks M. Aneel Kumar and Sini Sony, GIS Experts, RTS, Oman for producing the map. 
in the study. The most speciose family was Serranidae $(S=20)$, followed by the Acanthuridae $(S=18)$, Labridae $(\mathrm{S}=18)$, and Pomacentridae $(\mathrm{S}=16)$ (Table 1). Nocturnal and cryptic families such as the Gobiidae, Blennidae, and Scorpaenidae were present, but not identified till the generic or species level due to difficulties with visual identification. Other habitats such as mangrove forests, sand and mud flats, and deeper rocky reefs should also be surveyed in order to develop complete checklists for this region. Based on Fishbase records and relevant literature, we observed 12 species that were not previously recorded from India $(6.5 \%$ of all species observed). Twenty two species were recorded in other literature as occurring in India as well as being observed during this study. These should be added to occurrence records in Fishbase. Most of the non-commercial species observed are recorded from the western coast of India for the first time. Previous records of these species in India are usually from the Lakshadweep, or the Andaman and Nicobar Islands.

\section{Discussion}

All of the new records came from families of fish that are small and not usually commercially exploited. The exception being species within the family Acanthuridae which are likely caught artisanally. None of the species within these families have been assessed for their IUCN Red List status (see IUCN 2011). Only a few families of marine fish have been assessed for Red List status. All species of angelfish (Pomacanthidae) observed have been assessed as Least Concern (LC), and also the species of Butterflyfish (Chaetodontidate), with the exception of Chaetodon andamanensis which is Data Deficient (DD) (Myers \& Pratchett 2010). Fishbase lists this species as ocurring in India, Indonesia, and the Maldives. Studies of this species would be of conservation value as there is little data to understand its current conservation status. Both parrotfish (Scaridae) species observed were in the Least Concern category. All wrasses (Labridae) observed were of Least Concern status, with the exception of the Humphead Wrasse Cheilinus undulatus, which is listed as Endangered (EN) (Russell 2004). In this study, this species was observed in low numbers (Sluka \& Lazarus 2005). In another study, Nair (2004) reports on a specimen landed at Cochin Fisheries Harbour. This species is sought after in the live fish food trade and in

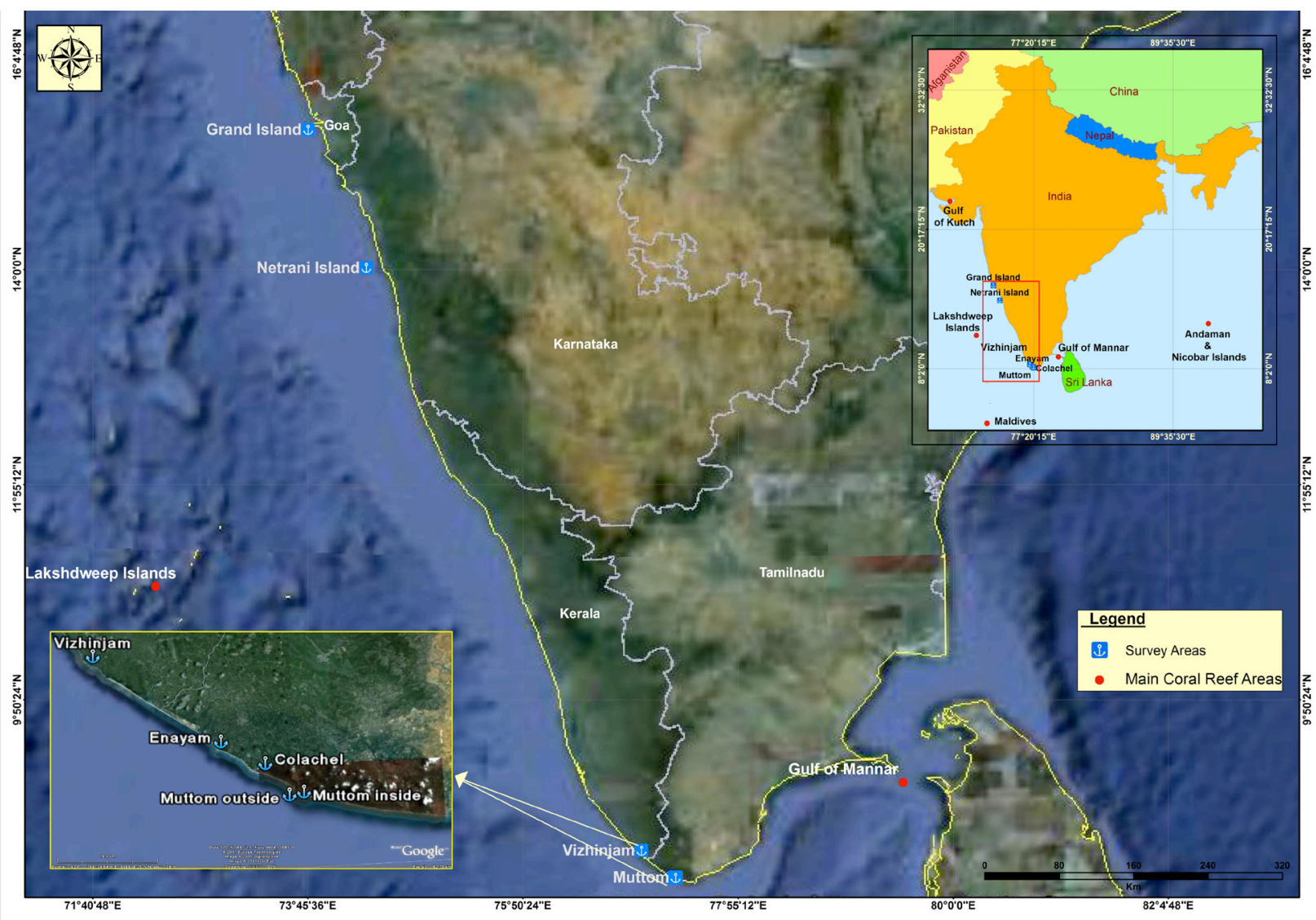

Image 1. Location of study sites along the west coast of India 
many locations it is severely overfished. One species of shark Nebrius ferrugineus (Tawny Nurse Shark) was observed. This species is Vulnerable in the IUCN Red List (Pillans 2003).

Twenty serranids (Serranidae) were observed, 18 of these assessed for Red List status, with 10 in the LC category. Species that were DD included Aethaloperca rogaa (Heemstra et al. 2008a), Epinephelus erythrurus (Heemstra et al. 2008b), and E. faveatus (Russell et al. 2008). The first species is a widespread Indo-Pacific species, but usually locally not very abundant. It is also not often caught in commercial fisheries. Fishbase states that nothing has been published on the biology of E. erythrurus and so this represents an opportunity to remedy its $\mathrm{DD}$ status. The distribution of E. faveatus is currently only known from southern India, Sri Lanka and Indonesia, so researchers in this area could contribute to a better understanding of its biology. Three species of serranid were Near Threatened (NT): E. coioides (Cornish \& Harmelin-Vivien 2004), E. diacanthus (Sadovy et al. 2008), and E. malabaricus (Cornish 2006). The distribution of $E$. diacanthus is restricted to the continental northern Indian Ocean. Effort should be put into understanding fishing pressure on this species and developing conservation measures to halt overfishing. The other two species are large with maximum sizes over $1 \mathrm{~m}$ and weighing $150 \mathrm{~kg}$, and heavily targeted globally for food. Two species observed were listed as Vulnerable (VU): E. lanceolatus (Man \& Chuen 2006) and Plectropomus areolatus (Thierry et al. 2008). Both species are large, with the former reaching $2.7 \mathrm{~m}$ in length and $400 \mathrm{~kg}$. For all grouper species, fishery regulations and implementation of conservation measures, including marine protected areas should be implemented.

\section{REFERENCES}

Allen, G.R. (1991). Damselfishes of The World. MERGUS Publishers, Melle, Germany, 271pp.

Allen, G.R. (1997). Marine Fishes of Tropical Australia and South-East Asia. Western Australia Museum, Perth, 292pp.

Allen, G.R., R. Steene \& M. Allen (1998). A Guide to Angelfishes \& Butteflyfishes. Odyssey Publishing/Tropical Reef Research, Perth, Australia, 250pp.

Anand, P.E.V. \& N.G.K. Pillai (2002). Habitat distribution and species diversity of coral reef fishes in the reefslope of the Kavaratti atoll, Lakshadweep, India. Journal of the Marine Biological Association of India 45(1): 88-98.

Anand, P.E.V. \& N.G.K. Pillai (2005). Community organization of coral reef fishes in the rubble sub-habitat of Kavaratti Atoll, Lakshadweep, India. Journal of the Marine Biological Association of India 47(1): 77-82.

Cornish, A. (Grouper \& Wrasse Specialist Group) (2006). Epinephelus malabaricus. In: IUCN 2012. IUCN Red List of Threatened Species. Version 2012.2. <www.iucnredlist.org>. Downloaded on 18 January
2013

Cornish, A. \& M. Harmelin-Vivien (Grouper \& Wrasse Specialist Group) (2004). Epinephelus coioides. In: IUCN 2012. IUCN Red List of Threatened Species. Version 2012.2. <www.iucnredlist.org>. Downloaded on 18 January 2013

De Bruin, G.H.P., B.C. Russel \& A. Bogusch (1994). FAO Species Identification Field Guide for Fishery Purposes. The marine fishery resources of Sri Lanka. FAO, Rome.

Fischer, W. \& G. Bianchi (eds.) (1984). FAO Species Identification Sheets for Fishery Purposes. Western Indian Ocean (Fishing Area 51). FAO, Rome.

Heemstra, P.C., M. Samoilys, A. Cornish \& A.S. Cabanban (2008). Aethaloperca rogaa. In: IUCN 2012. IUCN Red List of Threatened Species. Version 2012.2. <www.iucnredlist.org>. Downloaded on 18 January 2013.

Heemstra, P.C., L. Rocha, J.H. Choat, M.T. Craig, A.A. Bertoncini \& B. Ferreira (2008b). Epinephelus erythrurus. In: IUCN 2012. IUCN Red List of Threatened Species. Version 2012.2. <www.iucnredlist.org>. Downloaded on 18 January 2013.

IUCN (2011). The IUCN Red List of Threatened Species. Version 2011.2. <http://www.iucnredlist.org>. Downloaded on 1 October 2012.

James, P.S.B.R., S. Lazarus \& G. Arumugam (1994). The present status of 'major perch' fisheries in India. Central Marine Fisheries Research Institute Bulletin 47: 1-9.

James, P.S.B.R., S. Lazarus \& C.S.G. Pilllai (1988). On the availability of tuna live-bait fishes at Vizhinjam. Marine Fisheries Inforfmation Series: Technical Extension Series 84: 8-10.

James, P.S.B.R., V.S. Murty \& P. Nammalwar (1996). Groupers and snappers of India: biology and exploitation, pp. 106-136. In: Arreguin-Sanchez, F., J.L. Munro, M.C. Balgos \& D. Pauly (eds.) Biology, Fisheries and Culture of Tropical Groupers and Snappers. ICLARM Conference Proceedings 48, 449pp.

Jones, S. (1969). Catalogue of fishes from the Laccadive archipelago in the reference collections of the Central Marine Fisheries Research Institute. Central Marine Fisheries Research Institute Bulletin No. 8. 32pp.

Jones, S. \& M. Kumaran (1980). Fishes of the Laccadive Archipeligo. The Nature Conservation and Aquatic Science Service. Trivandrum, India, 760pp.

Kuiter, R.H. (1998). Fishes of the Maldives. Atoll Editions, Australia, 257pp.

Kuthalingam, M.D.K., M.S. Rajagopal, P.V. Sreenivasan, N. Radhakrishnan, S. Lazarus \& P. Livingston (1973). On some interesting fishes from the southwest coast of India. Journal of the Marine Biological Association of India 15: 877-882.

Kuthalingam, M.D.K., P.V. Sreenivasan \& S. Lazarus (1979). Four new records of fishes from Indian seas. Matsya 5: 42-46.

Madhu, R. \& K. Madhu (2007). Occurrence of anenomefishes and host sea anenomes in Andaman and Nicobar Islands. Journal of the Marine Biological Association of India 49(2): 118-126.

Man, C.S. \& N.W. Chuen (Grouper \& Wrasse Specialist Group) (2006). Epinephelus lanceolatus. In: IUCN 2012. IUCN Red List of Threatened Species. Version 2012.2. <www.iucnredlist.org>. Downloaded on 18 January 2013.

Murty, V.S. (1969). Catalogue of fishes (excluding from the Laccadives) in the reference collections of the Central Marine Fisheries Research Institute. Central Marine Fisheries Research Institute Bulletin No. 10, 36pp.

Murty, V.S., K. Kumaran \& R.S. Lalmohan (1987). Resources of ornamental fishes, pp. 46-64. In: Suseelan, C. (ed.) Marine Living Resources of The Union Territory of Lakshadweep - An Indicative Survey with Suggestions for Development. Central Marine Fisheries Research Institute Bulletin 43, 256pp.

Murty, V.S. (2002). Marine ornamental fish resources of Lakshadweep. Central Marine Fisheries Research Institute Special Publication No. 72, 134pp.

Myers, R. \& M. Pratchett (2010). Chaetodon andamanensis. In: IUCN 2012. IUCN Red List of Threatened Species. Version 2012.2. <www. iucnredlist.org>. Downloaded on 18 January 2013. 
Table 1. Fish species observed in nearshore rocky/coral habitats of western India. An F in the Records column indicates this species recorded as present in India by the Fishbase database (www.fishbase.org) as of 26 April 2012. Other references which establish fish distribution records for India are given below the table. Data with NR in the records column indicate new records for India ( $n=12$ ). IUCN status is given as LC $=$ Least Concern, DD = Data Deficient, NT = Near Threatened, VU = Vulnerable, EN $=$ Endangered, and NA $=$ Not Assessed.

\begin{tabular}{|c|c|c|c|c|c|}
\hline Species & Record & IUCN & S. India & Netrani & Goa \\
\hline \multicolumn{6}{|l|}{ Ginglymostomatidae } \\
\hline Nebrius ferrugineus & $\mathrm{F}$ & VU & & & $x$ \\
\hline \multicolumn{6}{|l|}{ Muraenidae } \\
\hline Gymnomuraena zebra & $\mathrm{F}$ & NA & $\mathrm{x}$ & & \\
\hline Gymnothorax favagineus & $\mathrm{F}$ & NA & $x$ & $x$ & $x$ \\
\hline G. flavimarginatus & $\mathrm{F}$ & NA & $\mathrm{x}$ & $\mathrm{x}$ & $x$ \\
\hline G. javanicus & $\mathrm{F}$ & NA & $x$ & & \\
\hline G. meleagris & $\mathrm{F}$ & NA & $x$ & & $x$ \\
\hline G. pictus & $\mathrm{F}$ & NA & $x$ & $x$ & \\
\hline \multicolumn{6}{|l|}{ Holocentridae } \\
\hline Myripristis botche & $\mathrm{F}$ & NA & $x$ & & \\
\hline $\begin{array}{l}\text { Sargocentron } \\
\text { caudimaculatum }\end{array}$ & $\mathrm{F}$ & NA & $x$ & & \\
\hline S. diadema & $\mathrm{F}$ & NA & $\mathrm{x}$ & & \\
\hline S. rubrum & $\mathrm{F}$ & NA & $x$ & $x$ & $x$ \\
\hline \multicolumn{6}{|l|}{ Fistulariidae } \\
\hline Fistularia commersonii & 6 & NA & $x$ & & \\
\hline F. petimba & $\mathrm{F}$ & NA & $x$ & & \\
\hline \multicolumn{6}{|l|}{ Scorpaenidae } \\
\hline Pterois miles & $2^{\mathrm{a}}$ & NA & $x$ & $x$ & $x$ \\
\hline P. mombasae & $\mathrm{F}$ & NA & $x$ & & \\
\hline \multicolumn{6}{|l|}{ Serranidae } \\
\hline Aethaloperca rogaa & $\mathrm{F}$ & DD & $x$ & $x$ & \\
\hline Cephalopholis argus & $\mathrm{F}$ & LC & $x$ & $x$ & \\
\hline C. formosa & $\mathrm{F}$ & LC & $x$ & $x$ & $x$ \\
\hline C. miniata & $\mathrm{F}$ & LC & $x$ & & \\
\hline C. sonnerati & $\mathrm{F}$ & LC & $x$ & $x$ & \\
\hline Diploprion bifasciatum & $\mathrm{F}$ & NA & $x$ & & \\
\hline Epinephelus areolatus & $\mathrm{F}$ & LC & & $x$ & \\
\hline E. coeruleopunctatus & $\mathrm{F}$ & LC & $x$ & & \\
\hline E. coioides & $\mathrm{F}$ & NT & $x$ & $x$ & $x$ \\
\hline E. diacanthus & $\mathrm{F}$ & NT & $x$ & $x$ & $x$ \\
\hline E. erythrurus & $\mathrm{F}$ & DD & $x$ & $x$ & $x$ \\
\hline E. fasciatus & $\mathrm{F}$ & LC & $x$ & & \\
\hline E. faveatus & $\mathrm{F}$ & DD & $x$ & & \\
\hline E. flavocaeruleus & $\mathrm{F}$ & LC & $x$ & & \\
\hline E. lanceolatus & $\mathrm{F}$ & Vu & & $x$ & \\
\hline E. longispinis & $\mathrm{F}$ & LC & $x$ & $x$ & \\
\hline E. malabaricus & $\mathrm{F}$ & NT & $x$ & $x$ & \\
\hline E. tukula & $\mathrm{F}$ & LC & $x$ & & $x$ \\
\hline Plectropomus areolatus & $\mathrm{F}$ & Vu & $x$ & & \\
\hline $\begin{array}{l}\text { Pseudanthias } \\
\text { squamipinnis }\end{array}$ & $\mathrm{F}$ & NA & $\mathrm{x}$ & & \\
\hline
\end{tabular}

\begin{tabular}{|c|c|c|c|c|c|}
\hline Species & Record & IUCN & S. India & Netrani & Goa \\
\hline \multicolumn{6}{|l|}{ Priacanthidae } \\
\hline Priacanthus hamrur & $\mathrm{F}$ & NA & $x$ & & \\
\hline \multicolumn{6}{|l|}{ Apogonidae } \\
\hline Apogon aureus & 6 & NA & $x$ & $x$ & \\
\hline A. hyalosoma & 6 & NA & $x$ & & \\
\hline A. pseudotaeniatus & 8 & NA & $x$ & & \\
\hline A. thermalis & NR & NA & $x$ & & \\
\hline Rhabdamia gracilis & $\mathrm{F}$ & NA & $x$ & & \\
\hline \multicolumn{6}{|l|}{ Rachycentridae } \\
\hline Rachycentron canadum & $\mathrm{F}$ & NA & & $x$ & \\
\hline \multicolumn{6}{|l|}{ Echeneidae } \\
\hline Echeneis naucrates & $\mathrm{F}$ & NA & $x$ & & $x$ \\
\hline \multicolumn{6}{|l|}{ Carangidae } \\
\hline Caranx heberi & $\mathrm{F}$ & NA & & $x$ & \\
\hline C. melampygus & $\mathrm{F}$ & NA & $x$ & & \\
\hline Gnathanodon speciosus & $\mathrm{F}$ & NA & $x$ & & \\
\hline Trachinotus baillonii & $\mathrm{F}$ & NA & $x$ & & \\
\hline \multicolumn{6}{|l|}{ Leiognathidae } \\
\hline Leiognathus splendens & $\mathrm{F}$ & NA & $x$ & & $x$ \\
\hline \multicolumn{6}{|l|}{ Lutjanidae } \\
\hline Aprion virescens & $\mathrm{F}$ & NA & $x$ & & \\
\hline $\begin{array}{l}\text { Lutjanus } \\
\text { argentimaculatus }\end{array}$ & $\mathrm{F}$ & NA & $x$ & $x$ & \\
\hline L. bohar & $\mathrm{F}$ & NA & $x$ & & \\
\hline L. decussatus & $\mathrm{F}$ & LC & $x$ & & \\
\hline L. fulvus & $\mathrm{F}$ & NA & $x$ & $x$ & $x$ \\
\hline L. gibbus & $\mathrm{F}$ & NA & $x$ & & \\
\hline L. kasmira & $\mathrm{F}$ & NA & $x$ & & \\
\hline L. lutjanus & $\mathrm{F}$ & NA & $x$ & $x$ & \\
\hline L. madras & $\mathrm{F}$ & NA & & $x$ & $x$ \\
\hline L. monostigma & $\mathrm{F}$ & NA & & $x$ & \\
\hline L. quinquelineatus & $\mathrm{F}$ & NA & $x$ & $x$ & \\
\hline L. rivulatus & $\mathrm{F}$ & NA & $x$ & $x$ & $x$ \\
\hline L. russelli & $\mathrm{F}$ & NA & & $x$ & $x$ \\
\hline L. vitta & $\mathrm{F}$ & NA & $x$ & $x$ & $x$ \\
\hline \multicolumn{6}{|l|}{ Caesonidae } \\
\hline Caesio cuning & $\mathrm{F}$ & NA & $x$ & $x$ & $x$ \\
\hline C. teres & $\mathrm{F}$ & NA & $x$ & & \\
\hline C. varilineata & $\mathrm{F}$ & NA & $x$ & $x$ & \\
\hline C. xanthonota & $\mathrm{F}$ & NA & $x$ & & \\
\hline Pterocaesio chrysozona & $\mathrm{F}$ & NA & $x$ & & \\
\hline P. tile & $\mathrm{F}$ & NA & $x$ & & \\
\hline \multicolumn{6}{|l|}{ Gerridae } \\
\hline Gerres erythrourus & $\mathrm{F}$ & NA & $x$ & & \\
\hline \multicolumn{6}{|l|}{ Haemulidae } \\
\hline $\begin{array}{l}\text { Diagramma pictum } \\
\text { cinerascens }\end{array}$ & $\mathrm{F}$ & NA & $x$ & & \\
\hline Plectorhincus schotof & $\mathrm{F}$ & NA & $x$ & $x$ & $x$ \\
\hline
\end{tabular}




\begin{tabular}{|c|c|c|c|c|c|}
\hline Species & Record & IUCN & S. India & Netrani & Goa \\
\hline Pomadasys furcatum & $\mathrm{F}$ & NA & $x$ & $x$ & $x$ \\
\hline P. gibbosus & $\mathrm{F}$ & NA & $x$ & $x$ & $x$ \\
\hline P. orientalis & 6 & NA & $x$ & & \\
\hline \multicolumn{6}{|l|}{ Sparidae } \\
\hline Rhabdosargus sarba & $\mathrm{F}$ & NA & $x$ & & \\
\hline \multicolumn{6}{|l|}{ Lethrinidae } \\
\hline Lethrinus harak & $\mathrm{F}$ & NA & $x$ & & \\
\hline L. nebulosus & $\mathrm{F}$ & NA & $x$ & & \\
\hline Monotaxis grandoculis & $\mathrm{F}$ & NA & $x$ & & \\
\hline \multicolumn{6}{|l|}{ Nemipteridae } \\
\hline Scolopsis auratus & $\mathrm{F}$ & NA & $x$ & & \\
\hline S. vosmeri & $\mathrm{F}$ & NA & $x$ & $x$ & $x$ \\
\hline \multicolumn{6}{|l|}{ Mullidae } \\
\hline Parupeneus barberinus & $\mathrm{F}$ & NA & $x$ & & \\
\hline P. cyclostomus & $\mathrm{F}$ & NA & $x$ & & \\
\hline P. indicus & $\mathrm{F}$ & NA & $x$ & $x$ & $x$ \\
\hline P. multifasciatus & $\mathrm{F}$ & NA & $x$ & & \\
\hline Upeneus vittatus & $\mathrm{F}$ & NA & $x$ & & \\
\hline \multicolumn{6}{|l|}{ Pempheridae } \\
\hline Pempheris schwenkii & NR & NA & & $x$ & $x$ \\
\hline P. vanicolensis & $\mathrm{F}$ & NA & $x$ & & \\
\hline \multicolumn{6}{|l|}{ Kyphosidae } \\
\hline Kyphosus vaigiensis & $\mathrm{F}$ & NA & $x$ & & \\
\hline \multicolumn{6}{|l|}{ Ephippidae } \\
\hline Platax orbicularis & 6 & NA & $x$ & & \\
\hline \multicolumn{6}{|l|}{ Monodactylidae } \\
\hline Monodactylus argenteus & $\mathrm{F}$ & NA & $x$ & & $x$ \\
\hline \multicolumn{6}{|l|}{ Scatophagidae } \\
\hline Scatophagus argus & $\mathrm{F}$ & NA & $x$ & & \\
\hline \multicolumn{6}{|l|}{ Chaetodontidae } \\
\hline Chaetodon andamanensis & $\mathrm{F}$ & DD & & & \\
\hline C. auriga & $\mathrm{F}$ & LC & $x$ & $x$ & \\
\hline C. collare & $\mathrm{F}$ & LC & $x$ & $x$ & $x$ \\
\hline C. decussatus & $\mathrm{F}$ & LC & $x$ & $x$ & $x$ \\
\hline C. lineolatus & 6 & LC & $x$ & & \\
\hline C. Iunula & $\mathrm{F}$ & LC & $x$ & & \\
\hline C. vagabundus & 1 & LC & $x$ & & \\
\hline Forcipiger longirostris & $\mathrm{F}$ & LC & $x$ & & \\
\hline Heniochus acuminatus & $\mathrm{F}$ & LC & $x$ & $x$ & $x$ \\
\hline \multicolumn{6}{|l|}{ Pomacanthidae } \\
\hline Apolemichthys xanthurus & $\mathrm{F}$ & LC & $x$ & & \\
\hline Centropyge flavipectoralis & 5 & LC & $x$ & & \\
\hline Pomacanthus annularis & $\mathrm{F}$ & LC & $x$ & $x$ & $x$ \\
\hline P. imperator & $\mathrm{F}$ & LC & $x$ & & \\
\hline P. semicirculatus & $\mathrm{F}$ & LC & $x$ & & \\
\hline \multicolumn{6}{|l|}{ Cirrhitidae } \\
\hline Cirrhitichthys bleekeri & $\mathrm{F}$ & NA & $x$ & & \\
\hline C. oxycephalus & NR & NA & $x$ & & \\
\hline Pomacentridae & & & & & \\
\hline
\end{tabular}

\begin{tabular}{|c|c|c|c|c|c|}
\hline Species & Record & IUCN & S. India & Netrani & Goa \\
\hline Abudefduf bengalensis & $\mathrm{F}$ & NA & & $x$ & $x$ \\
\hline A. notatus & 6 & NA & $x$ & & \\
\hline A. septemfasciatus & $\mathrm{F}$ & NA & $x$ & & \\
\hline A. sordidus & $\mathrm{F}$ & NA & $x$ & $x$ & $x$ \\
\hline A. vaigiensis & $\mathrm{F}$ & NA & $x$ & $x$ & $x$ \\
\hline Amphiprion sebae & $\mathrm{F}$ & NA & $\mathrm{x}$ & & \\
\hline Chromis atripectoralis & $\mathrm{F}$ & NA & $x$ & $x$ & $x$ \\
\hline C. viridis & $\mathrm{F}$ & NA & $\mathrm{x}$ & & \\
\hline Chrysiptera biocellata & $\mathrm{F}$ & NA & $x$ & & \\
\hline C. leucopoma & 1 & NA & $x$ & & \\
\hline C. unimaculata & $\mathrm{F}$ & NA & $x$ & $x$ & $x$ \\
\hline Dascyllus trimaculatus & $\mathrm{F}$ & NA & $x$ & & \\
\hline $\begin{array}{l}\text { Neopomacentrus } \\
\text { cyanamos }\end{array}$ & 4 & NA & $\mathrm{x}$ & & $x$ \\
\hline Pomacentrus caeruleus & 4 & NA & $x$ & & \\
\hline P. chrysurus & NR & NA & $\mathrm{x}$ & & \\
\hline P. indicus & NR & NA & $x$ & & \\
\hline \multicolumn{6}{|l|}{ Labridae } \\
\hline $\begin{array}{l}\text { Anampses } \\
\text { caeruleopunctatus }\end{array}$ & $\mathrm{F}$ & LC & $x$ & & \\
\hline Bodianus axillaris & 7 & LC & $x$ & & \\
\hline B. diana & 7 & LC & $x$ & & \\
\hline B. neilli & $\mathrm{F}$ & LC & $x$ & $x$ & \\
\hline Cheilinus undulatus & $\mathrm{F}$ & EN & $x$ & & \\
\hline Coris formosa & $\mathrm{F}$ & LC & $x$ & $\mathrm{x}$ & \\
\hline Gomphosus caeruleus & $\mathrm{F}$ & LC & $x$ & & \\
\hline Halichoeres hortulanus & $\mathrm{F}$ & LC & $x$ & & \\
\hline H. marginatus & $\mathrm{F}$ & LC & $x$ & & \\
\hline H. nebulosua & $\mathrm{F}$ & LC & $x$ & & \\
\hline H.nigrescens & $\mathrm{F}$ & LC & & $x$ & $x$ \\
\hline H. zeylonicus & $\mathrm{F}$ & LC & $x$ & & \\
\hline Labroides dimidiatus & $\mathrm{F}$ & LC & $x$ & $x$ & $x$ \\
\hline Stethojulis strigiventer & $\mathrm{F}$ & LC & & $x$ & \\
\hline S. trilineata & $\mathrm{F}$ & LC & $x$ & & \\
\hline Thalassoma hardwicke & $\mathrm{F}$ & LC & $x$ & & \\
\hline T. jansenii & $\mathrm{F}$ & LC & $x$ & & \\
\hline T. lunare & $\mathrm{F}$ & LC & $x$ & $x$ & $x$ \\
\hline \multicolumn{6}{|l|}{ Scaridae } \\
\hline Scarus ghobban & $\mathrm{F}$ & LC & $x$ & $x$ & $x$ \\
\hline Scarus rubrioviolaceus & $\mathrm{F}$ & LC & $x$ & $x$ & \\
\hline \multicolumn{6}{|l|}{ Blenniidae } \\
\hline $\begin{array}{l}\text { Plagiotremus } \\
\text { rhinorhyncus }\end{array}$ & $\mathrm{F}$ & NA & $x$ & & \\
\hline \multicolumn{6}{|l|}{ Siganidae } \\
\hline Siganus canaliculatus & $\mathrm{F}$ & NA & & $x$ & $x$ \\
\hline S. javas & $\mathrm{F}$ & NA & $x$ & $x$ & $x$ \\
\hline S. guttatus & $\mathrm{F}$ & NA & $x$ & & \\
\hline \multicolumn{6}{|l|}{ Zanclidae } \\
\hline Zanclus cornutus & $\mathrm{F}$ & NA & $x$ & $x$ & $x$ \\
\hline \multicolumn{6}{|l|}{ Acanthuridae } \\
\hline Acanthurus auranticavus & NR & NA & & $x$ & $x$ \\
\hline
\end{tabular}




\begin{tabular}{|c|c|c|c|c|c|}
\hline Species & Record & IUCN & S. India & Netrani & Goa \\
\hline A. bariene & NR & NA & $x$ & & \\
\hline A. leucocheilos & NR & NA & $\mathrm{x}$ & $\mathrm{x}$ & \\
\hline A. leucosternon & $\mathrm{F}$ & NA & $\mathrm{x}$ & & \\
\hline A. lineatus & $\mathrm{F}$ & NA & $x$ & & \\
\hline A. mata & $\mathrm{F}$ & NA & $x$ & $x$ & \\
\hline A. nigricauda & 6 & NA & $x$ & & \\
\hline A. tennentii & $\mathrm{F}$ & NA & $x$ & & \\
\hline A. thompsoni & $\mathrm{F}$ & NA & & $x$ & \\
\hline A. triostegus & $\mathrm{F}$ & NA & $x$ & & \\
\hline A. xanthopterus & $\mathrm{F}$ & NA & & & \\
\hline Ctenochaetus binotatus & NR & NA & $x$ & & \\
\hline C. striatus & $\mathrm{F}$ & NA & $x$ & & \\
\hline C. strigosus & $\mathrm{F}$ & NA & $x$ & $x$ & \\
\hline Naso brachycentron & $\mathrm{F}$ & NA & $x$ & & \\
\hline N. brevirostris & $\mathrm{F}$ & NA & $x$ & & \\
\hline N. hexacanthus & NR & NA & $x$ & & \\
\hline Zebrasoma desjardini & 1 & NA & $x$ & & \\
\hline \multicolumn{6}{|l|}{ Sphyraenidae } \\
\hline Sphyraena barracuda & $\mathrm{F}$ & NA & $x$ & & \\
\hline S. jello & $\mathrm{F}$ & NA & $x$ & & \\
\hline \multicolumn{6}{|l|}{ Balistidae } \\
\hline Balistoides viridescens & 1 & NA & $x$ & & \\
\hline Odonus niger & $\mathrm{F}$ & NA & $x$ & $x$ & $x$ \\
\hline $\begin{array}{l}\text { Pseudobalistes } \\
\text { flavimarginatus }\end{array}$ & $\mathrm{F}$ & NA & $x$ & & \\
\hline P. fuscus & $\mathrm{F}$ & NA & $x$ & & \\
\hline Sufflamen chrysopterus & 6 & NA & $x$ & & \\
\hline S. fraenatum & 3 & LC & $x$ & $x$ & $x$ \\
\hline \multicolumn{6}{|l|}{ Monocanthidae } \\
\hline Pervagor janthinosoma & NR & NA & $x$ & & \\
\hline \multicolumn{6}{|l|}{ Ostraciidae } \\
\hline Ostracion cubicus & $\mathrm{F}$ & NA & $x$ & & \\
\hline Tetrosomas concatenatus & NR & NA & & $x$ & \\
\hline \multicolumn{6}{|l|}{ Tetradontidae } \\
\hline Arothron hispidus & $\mathrm{F}$ & NA & $x$ & & \\
\hline A. immaculatus & $\mathrm{F}$ & NA & $x$ & & \\
\hline A. meleagris & $\mathrm{F}$ & NA & & $x$ & \\
\hline A. nigropunctatus & $\mathrm{F}$ & NA & & $x$ & $x$ \\
\hline Chelonodon patoca & $\mathrm{F}$ & NA & & & $x$ \\
\hline \multicolumn{6}{|l|}{ Diodontidae } \\
\hline Diodon holocanthus & $\mathrm{F}$ & NA & $x$ & & \\
\hline D. hystrix & $\mathrm{F}$ & NA & $x$ & $x$ & $x$ \\
\hline D. liturosus & 6 & NA & $x$ & $x$ & \\
\hline
\end{tabular}

$\left({ }^{1}\right.$ Jones 1969; ${ }^{2}$ Murty $1969 ;{ }^{3}$ Fischer \& Bianchi $1984 ;{ }^{4}$ Allen $1991 ;{ }^{5}$ Allen et al. 1998; ${ }^{6}$ Rao et al. 2000; ${ }^{7}$ Sluka \& Lazarus 2004; ${ }^{8}$ Suresh \& Thomas 2007)-. ${ }^{a}$ Fishbase records Pterois volitans as present in India. However, the range of this species as given in the Fishbase record indicates otherwise and is likely a mistake as $P$. volitans is replaced by the very similar $P$. miles in the western Indian Ocean.
Nair, R.J. (2004). Note on a rare coral fish Cheilinus undulatus (Riippell, 1835). Journal of the Marine Biological Association of India 46(2): 234-236.

Pillans, R. (SSG Australia \& Oceania Regional Workshop, March 2003) (2003). Nebrius ferrugineus. In: IUCN 2012. IUCN Red List of Threatened Species. Version 2012.2. <www.iucnredlist.org>. Downloaded on 18 January 2013.

Rajaram, R. \& T. Nedumaran (2009). Ichthyofaunal diversity in Great Nicobar Biosphere Reserve, Bay of Bengal. Journal of Threatened Taxa 1(3): 166-169.

Rao, D.V., K. Devi \& P.T. Rajan (2000). An account of ichthyofauna of Andaman \& Nicobar Islands, Bay of Bengal. Records of the Zoological Survey of India Occasional Papers No. 178: 1-434.

Russell, B. (Grouper \& Wrasse Specialist Group) (2004). Cheilinus undulatus. In: IUCN 2012. IUCN Red List of Threatened Species. Version 2012.2. <www.iucnredlist.org>. Downloaded on 18 January 2013.

Russell, B., M. Samoilys, R. Myers, P.C. Heemstra, S. Fennessy \& A.S. Cabanban (2008). Epinephelus faveatus. In: IUCN 2012. IUCN Red List of Threatened Species. Version 2012.2. <www.iucnredlist.org>. Downloaded on 18 January 2013.

Sadovy, Y., D. Pollard, B. Russell \& P.C. Heemstra (2008). Epinephelus diacanthus. In: IUCN 2012. IUCN Red List of Threatened Species. Version 2012.2. <www.iucnredlist.org>. Downloaded on 18 January 2013.

Sluka, R.D. \& S. Lazarus (2004). Grouper and wrasse biodiversity along the west coast of India. Newsletter of the IUCN Grouper \& Wrasses Specialist Group 8: 6-10.

Sluka, R.D. \& S. Lazarus (2005). Humphead Wrasse (Cheilinus undulatus) rare on the west coast of India. Journal of the Marine Biological Association of the United Kingdom 85: 1293-1294.

Sluka, R.D. \& S. Lazarus (2006). Groupers and wrasses of Minicoy Island, Lakshadweep, India. Newsletter of the IUCN Grouper \& Wrasses Specialist Group 9: 4-5.

Sluka, R.D. \& S. Lazarus (2009). Reefs of the India's west coast. Reef Encounter 37: 18.

Sluka, R.D. \& S. Lazarus (2010). Grouper (Pisces: Serranidae) relative abundance and diversity on the west coast of India. Marine Biodiversity Records 3: e71 doi:10.1017/S1755267210000606.

Sluka, R.D., A.G. Mary \& S. Lazarus (2012). The biota of shallow, rocky reefs on the west coast of India. CORDIO Status Report 2011. http:// www.cordioea.org/status-report-2011/

Sreenivasan, P.V. \& S. Lazarus (1973). On two new distributional records of fishes from the Indian coast. Journal of the Marine Biological Association of India 15: 886-888.

Suresh, G.K. \& P.A. Thomas (2007). Three new records of Apogonids (Order: Perciformes, sub order: Percoidei) from the Indian Seas. Journal of the Marine Biological Association of India 49(1): 105108.

Thierry, C., Y. Sadovy \& B. Yeeting (2008). Plectropomus areolatus. In IUCN 2012. IUCN Red List of Threatened Species. Version 2012.2. <www.iucnredlist.org>. Downloaded on 18 January 2013.

Thomas, S., M.P. Sreeram, V.S. Kakati, M.K. Manisseri \& R.M. George (2011). Coral fish diversity in Netrani waters off Murudeshwar Karnataka, south India. Indian Journal of Fisheries 58(1): 45-51.

Zacharia, P.U., P.K. Krishnakumar, A.P. Dineshbabu, K. Vijayakumaran, P. Rohit, S. Thomas, G. Sasikumar, P. Kaladharan, R.N. Durgekar \& K.S. Mohamed (2008). Species assemblage in the coral reef ecosystem of Netrani Island off Karnataka along the southwest coast of India. Journal of the Marine Biological Association of India 50(1): 87-97. 